\title{
Praxiszertifzierung
}

\section{Qualität und Fortbildung für das Patientenwohl}

\author{
Niedergelassene Ärzte müssen nicht, aber sie können die Qualität \\ ihrer Praxen zertifizieren lassen. Einer, der Wert darauf legt, dass \\ jede Angestellte aufgabenspezifische Fortbildungen wahrnimmt, \\ ist der Urologe Dr. Wolfgang Lessel aus Magdeburg.
}

$\mathrm{D}$ ie Praxis von Dr. Wolfgang Lessel wurde erstmals vor zwei Jahren nach QEP (Qualität und Entwicklung in Praxen) zertifiziert. An manchen Tagen kommen bis zu 120 Patienten in die uroonkologische Schwerpunktpraxis. Das geht nur, wenn alles reibungslos läuft, sich der Arzt und seine sieben Medizinischen Fachangestellten (MFA) blind verstehen. „Da wir täglich merken, dass unsere Arbeit von den Patienten anerkannt wird, ist die Praxis ein bisschen wie unsere eigene. Wir arbeiten nicht nur sehr gern hier, wir fühlen uns auch für den ganzen $\mathrm{Ab}$ lauf mitverantwortlich", erklärt Diana Hauck, die leitende MFA und erste Ansprechpartnerin für den Chef und Praxisinhaber.

„Auch in einem kleinen Team ist es wichtig, Verantwortung festzulegen, um einen optimalen Ablauf zu gewährleisten“, ist Lessel überzeugt. Je größer das Team ist, desto wichtiger ist eine klare Aufgabenverteilung. „Ich kann Aufgaben delegieren und gleichzeitig Verantwortung abgeben. Durch ständige Rückinfomation ist der Gesamtüberblick jederzeit gewährleistet", so Lessel.

Trotz bewährter und guter Praxisorganisation haben sich Arzt und Team gemeinsam für die Zertifizierung und die damit gut anderthalbjährige Vorbereitung entschieden. Die einzelnen Aufgaben auf dem Weg dorthin teilten die MFA unter sich auf, wobei Jeanette König, die das geforderte Handbuch anlegte, den größten Part übernahm. Heute ist im Handbuch jeder einzelne Arbeitsschritt, jedes Aufgabengebiet aus- führlich beschrieben. „Das erleichtert die Arbeit besonders bei einer Urlaubsoder Krankheitsvertretung. Die Einarbeitung in das spezielle Beschäftigungsgebiet wird deutlich leichter", meint Jeanette König.

Team und Arzt sind sich einig, dass sich nach intensiver Diskussion um das neue Handbuch viele neue Ideen wie von selbst entwickelt haben. „Wer über den Arbeitsalltag in Ruhe nachdenkt, findet auch Ecken und Kanten, an denen es klemmt, macht sich selbst den Blick frei für Neues. Das ist im täglichen Stress sonst fast unmöglich“, sagt Lessel.

\section{Patienten profitieren von Spezialsprechstunden ...}

Neu sind beispielsweise gesonderte Spezialsprechstunden für Patienten mit Blasen- oder andrologischen Problemen. Der Vorteil: „Wir können Betroffenen schneller und gezielter Termine vergeben und haben zugleich die oft zeitaufwändigen Gespräche und Untersuchungen aus der Normalsprechstunde herausgelöst“, erklärt Peggy Stark. Gleiches geschah bei den chirurgischen Eingriffen. Lessel operiert jetzt nur noch mittwochs. Eine Sprechstunde gibt es an diesem Tage nicht mehr. „Früher hat Doktor Lessel immer den Spagat zwischen Sprechstunde und OP versucht", berichtet Diana Hauck. Dies habe zu unnötigen Wartezeiten für Patienten und Leerlaufzeiten im OP- und Sprechstundenbereich geführt.

Im Laufe des Zertifizierungsverfahrens haben sich die Angestellten zusätzlich für eine tägliche Dienstbesprechung vor
Sprechstundenbeginn entschieden. „Wir machen das aus freien Stücken, weil wir gemerkt haben, dass der Tag stressfreier verläuft, wenn wir morgens anstehende Aufgaben besprechen, notwendige Behandlungsabläufe einzelner Patienten optimieren“, erklärt Jeanette König.

\section{... und die Mitarbeiter von den Weiterbildungen}

Darüber hinaus findet mindestens einmal im Quartal eine große Teambesprechung statt, während der die gesamte Mannschaft die vergangenen Wochen Revue passieren lässt, aber vor allem künftige Aufgaben und Anforderungen besprochen und Probleme geklärt werden. Hier wird auch über Weiterbildungen entschieden. „Ich will, dass meine Angestellten fachlich top bleiben", begründet Lessel. Alle Frauen qualifizieren sich zur „onkologisch weitergebildeten Fachschwester". Arzt und Fachschwestern nehmen jährlich an Urologenkongressen teil, und die Mitarbeiterinnen absolvieren spezielle Kurse für ihre Ressorts. Jede der Mitarbeiterinnen kommt auf etwa zehn Weiterbildungsveranstaltungen pro Jahr.

Lessel engagiert sich im uroonkologischen Arbeitskreis Sachsen-Anhalt, dem Vertragsärzte und Kliniker angehören. Ziel ist die gute Zusammenarbeit zwischen ambulant und stationär tätigen Urologen. „Wir bekommen ständige Rückinformationen über die Behandlungsabläufe unserer stationären Patienten und können die Therapie so nahtlos fortsetzen." Als Mitglied des Prostatakarzinomzentrums legt der Urologe zudem großen Wert auf eine bessere Versorgung uroonkologischer Patienten, besonders bei ambulanten Chemotherapien, um die Qualität der Versorgung trotz Mehrbelastung sichern zu können. Auch dabei helfe die alle drei Jahre anstehende Re-Zertifizierung. Sie ist in der fast papierlosen Praxis Garantie dafür, dass sich Alltagsgewohnheiten nur schwer einschleichen können.

zie 